\title{
Principles of Directed Electronic Energy Transfer
}

David L. Andrews* and Richard G. Crisp

Nanostructures and Photomolecular Systems

School of Chemical Sciences

University of East Anglia

Norwich NR4 7TJ

United Kingdom

d.1.andrews@uea.ac.uk

$\underline{\text { Table of Contents }}$

1 Introduction

2 Energy Transfer between Isolated Chromophore Pairs

3 Energy Transfer in Multi-Chromophore Environments

4 Directed Energy Transfer Systems

5 Future Applications

List of Abbreviations

QED,

Quantum electrodynamics;

RET,

Resonance energy transfer;

LARET,

Laser-assisted resonance energy transfer;

IVR,

Intramolecular vibrational relaxation;

$D$,

Donor;

A,

Acceptor;

$M$,

Auxiliary chromophore;

$\xi$

Chromophore index;

W,

Rate of energy transfer;

$F_{D}(\omega)$,

Donor emission spectrum;

$\sigma_{A}(\omega)$

Acceptor absorption cross-section; 
$\tau_{D}$

$\hbar \omega$,

$R_{\xi \xi^{\prime}}$,

$\kappa_{3}^{\xi \xi^{\prime}}$ and $\kappa_{1}^{\xi \xi^{\prime}}$

$\mu^{D}$,

$\mu^{A}$,

$\mathcal{E}$

$d^{\xi}$

$\omega^{\prime}$

$I\left(\omega^{\prime}\right)$,

$\boldsymbol{e}$,

$\mu^{M}$

$\alpha^{M}(\omega)$,
Donor radiative lifetime;

Energy transferred via RET;

Separation of the chromophores $\xi$ and $\xi^{\prime}$;

Orientation factors for energy transfer between $\xi$ and $\xi^{\prime}$;

Dipole moment of the transition from the excited to ground state in $D$;

Dipole moment of the transition from the ground to excited state in $A$;

Electric displacement vector of a static electric field;

Absolute difference between the magnitudes of the excited and ground state dipole-transition moments of the chromophore $\xi$;

Frequency of applied throughput radiation;

Intensity of applied throughput radiation;

Polarization vector of applied throughput radiation;

Static dipole-moment of $M$;

Dynamic polarizability (at frequency $\omega$ ) of $M$. 


\section{Introduction}

In non-homogeneous absorptive materials, the primary result of uv/visible absorption is the population of short-lived electronic excited states in individual molecules or chromophore sites. One or more transfers of electronic excitation energy may occur between the initial absorption and eventual fluorescence emission events, commonly on an ultrafast timescale and with some associated losses such as vibrational dissipation. The routing of energy flow is determined by a sequence of transfer steps, beginning and ending at chromophores that differ either chemically or, if the chromophores are chemically equivalent, through local modifications in energy level structure arising from differences in electronic environment (e.g. bathochromic shifts). At the molecular scale, each elementary transfer step is a radiationless pairwise interaction, generally between an electronically excited donor and an electronically distinct acceptor which initially resides in its ground state. Förster [1] first demonstrated the Coulombic origin of this interaction, now known as resonance energy transfer (RET), also deriving its inverse sixth power dependence on the donoracceptor separation.

It is now known [2-7] that the Förster interaction is the short-range limit of a more general result given by a unified transfer theory - a theory that is valid over any distance, and which includes additional terms with inverse fourth power and inverse square dependences on separation. At large donor-acceptor separations, it emerges that the energy transfer is a radiative process involving the distinct emission and subsequent absorption of a photon. At shorter separations the radiationless process is exhibited. In each case, the efficiency of transfer depends on the extent of overlap between the emission spectrum of the donor and the absorption spectrum of the acceptor. If, however, chromophores are closer still - close enough for their wavefunctions to overlap (generally a separation of less than $0.4 \mathrm{~nm}$ ) - then a Dexter exchange mechanism comes into play [8]. This form of energy transfer involves a donor exchanging its excited electron with an unexcited one from an acceptor. Even if the donor and acceptor are separated beyond wavefunction overlap, electron exchange may still occur via a super-exchange mechanism [9-12], in which the 
coupling of the pair operates through a delocalization or mixing of the chromophore wavefunctions with those of bridging species (relay chromophores), connecting bonds or the local solvent cage. Energy transfer may thus be further directed between chromophores linked by suitable intermediaries.

On the optical irradiation of an optically dense system comprising a large number of chromophores, a number of pairwise interactions typically occur before the system enters in a stable state, and the energy transfer path accordingly comprises a series of short hops rather than one long one - largely as a result of the inverse sixth power distance dependence. Such multi-step resonance energy transfer might be expected to have the character of a random walk, as indeed occurs in homogeneous single-component systems. However, with suitable chromophore differentiation, functionally unidirectional transfer is exhibited in multichromophore systems, assisted by suitable chromophore disposition, and often the operation of a spectroscopic gradient. Through experimental studies involving photobleaching spectroscopy, it has recently been shown possible to trace the intricate form of such multi-step resonance energy transfer [13], allowing sequential mechanisms to be established in kinetically and spectroscopically complex systems. Whilst the principles that govern the directionality of energy flow in multichromophore systems is largely understood, other recent developments have identified new possibilities for effecting externally determined directional control. In particular it transpires that, by a variety of means, optical and electronic perturbations of light-harvesting systems can exert significant additional directing influences on the energy transit. The delineation and analysis of these novel mechanisms is the main subject of this chapter.

We begin in Section 2 by reviewing the theory underlying the fundamental mechanism for pairwise energy transfer, including modifications that result from the effects of applied electrical and optical fields. In Section 3 the general implications for multichromophore systems are explored, the principles then being illustrated by reference to biological and dendrimeric systems in Section 4. Also in Section 4, a model system utilizing these principles is developed, and it is shown how RET can be tailored, through the involvement of ancillary chromophores or non-resonant throughput radiation, to more efficiently direct energy flow. The chapter concludes in Section 5 with a discussion of the future prospects. 


\section{Energy Transfer between Isolated Chromophore Pairs}

To understand the multi-step energy transfer that typifies the operation of many complexes, it is appropriate to begin with the theory for the prototypical case of energy transfer between one specific excited chromophore and another in its ground state. This is discussed in the first sub-section - the following dealing with modifications to the basic process, including the influences of applied static and nonresonant radiation fields. A depiction of the three processes to be covered in this section is given in Figure 1.

\section{Fig. 1}

\subsection{Fundamental Theory for Energy Transfer in a Donor-Acceptor Pair}

The pairwise transfer of energy between two chromophores $D$ and $A$ can be described by the equation:

$$
D^{*}+A \longrightarrow D+A^{*}
$$

where $\xi^{*}$ denotes an excited state for chromophore $\xi$. Chromophore $D$ is designated the donor and $A$ the acceptor, though it should be emphasized that $D$ and $A$ may each adopt the alternative role in another part of a multi-step sequential transfer processes. For the description of the primary event (1), neither the mechanism for the excitation of $D$ nor the subsequent decay of $A$ comes into play; these are kinetically separable events. Using the Fermi Rule, the rate of energy transfer is determined from the square of a quantum amplitude given by the unified theory [14], and emerges as follows (based on the premise of electric dipole - electric dipole coupling); 


$$
W_{D A}=\frac{9}{8 \pi c^{2} \tau_{D}} \int F_{D}(\omega) \sigma_{A}(\omega) \omega^{2} \eta^{D A}\left(\omega, \boldsymbol{R}_{D A}\right) \mathrm{d} \omega
$$

Here $\sigma_{A}(\omega)$ is the absorption cross-section of the acceptor, $F_{D}(\omega)$ is the donor emission spectrum normalized to unity, and $\omega$ is the circular frequency associated with the transferred energy - the latter thus designated as $\hbar \omega$. The spectral overlap integral given by Eq. (2) is calculated by integration over a frequency range that completely encompasses the salient features of the donor emission and acceptor absorption spectra. Also in Eq. (2), $\tau_{D}$ is the donor radiative lifetime (a product of the measured fluorescence lifetime and the fluorescence quantum yield), $\boldsymbol{R}_{D A}=R_{D}-R_{A}$ is the vector separation of the chromophores, and $\eta^{D A}\left(\omega, \boldsymbol{R}_{D A}\right)$ is given by:

$$
\eta^{D A}\left(\omega, \boldsymbol{R}_{D A}\right)=\frac{c^{6}}{\omega^{6} R_{D A}^{6}}\left(\kappa_{3}^{D A}\right)^{2}+\frac{c^{4}}{\omega^{4} R_{D A}^{4}}\left[\left(\kappa_{3}^{D A}\right)^{2}-2 \kappa_{3}^{D A} \kappa_{1}^{D A}\right]+\frac{c^{2}}{\omega^{2} R_{D A}^{2}}\left(\kappa_{1}^{D A}\right)^{2}
$$

The quantity given by Eq. (3) represents the overall spatial dependence for the transfer mechanism, and as such comprises both translational and orientational elements. It is interesting how the distance dependence is tempered by the transfer energy - note that $c / \omega R_{D A}=\lambda / 2 \pi R_{D A}$, where the $\lambda$ is the wavelength corresponding to the transfer energy. Furthermore the orientation factors $\kappa_{3}^{D A}$ and $\kappa_{1}^{D A}$ in Eq. (3) are defined as;

$$
\boldsymbol{\kappa}_{q}^{D A}=\left(\hat{\boldsymbol{\mu}}^{D} \cdot \hat{\boldsymbol{\mu}}^{A}\right)-q\left(\hat{\boldsymbol{\mu}}^{D} \cdot \hat{\boldsymbol{R}}_{D A}\right)\left(\hat{\boldsymbol{\mu}}^{A} \cdot \hat{\boldsymbol{R}}_{D A}\right)
$$

with $\hat{\boldsymbol{\mu}}^{D}, \hat{\boldsymbol{\mu}}^{A}, \hat{\boldsymbol{R}}_{D A}$ being unit vectors in the direction of the dipole transition moments and the donor-acceptor separation vector. The $\kappa$ factors thus signify an orientational dependence determined by the detailed chromophore architecture. For certain angular dispositions of the displacement vector and dipole transition moments (for example if all are mutually orthogonal) the $\kappa$ factors vanish, obviating energy transfer by this 
mechanism. At the other extreme, optimization of the energy transfer is achieved when both transition moments are parallel or antiparallel to the separation vector [15]. Analysis of Eq. (2) indicates that the transfer rate is also strongly influenced by the separation and spectral features of the two chromophores, in addition to the relative orientation of the transition dipoles.

It may be observed that the detailed form delivered by the unified theory incorporates terms associated with both the commonly termed 'radiative' and 'radiationless' processes, through $\eta^{D A}\left(\omega, \boldsymbol{R}_{D A}\right)$. In optically dilute systems with chromophore spacings of about a hundred or a few hundred nanometers, all three terms in Eq. (3) are comparable in magnitude. However for transfer over longer distances (the far-zone, $R \gg \lambda / 2 \pi$, corresponding to distances well in excess of the wavelength for the transfer energy) the third term in Eq. (3) dominates and 'inverse square' radiative behavior $\left(W_{D A} \propto \kappa_{1}^{2} / R^{2}\right)$ is observed. Conversely, in the near-zone ( $R \ll \lambda / 2 \pi$, as is usual for energy transfer in optically dense condensed phase systems) Eq. (2) produces the familiar Förster rate;

$$
W_{D A}^{R E T}=\frac{9 c^{4} \kappa_{3}^{2}}{8 \pi \tau_{D} R_{D A}^{6}} \int F_{D}(\omega) \sigma_{A}(\omega) \omega^{-4} \mathrm{~d} \omega,
$$

with a direct dependence on $R^{-6}$ and $\kappa_{3}^{2}$. The latter near-zone behavior, usually termed 'radiationless', is by far the most significant for the near-neighbor transfers that occur in light-harvesting and allied materials.

\subsection{Influence of a Static Electric Field}

The rate of energy transfer between a donor and acceptor pair can be significantly modified by interaction with a static electric field [16]. The effect is a direct consequence of associated shifts in the electron distributions of the interacting chromophores. In detail it transpires that the mechanism for delivery of energy to the acceptor comprises four quantum pathways. The quantum amplitude for the overall 
transfer process is dominated by a term that exactly corresponds to the usual fieldindependent result, but it also comprises correction terms - the most significant of which entail linear coupling of the static field with either $D$ or $A$, and one other involves coupling of the static field at both $D$ and $A$. Moreover, when the static field engages with a transition, it is associated with different selection rules - which can be formally identified with those of a two-quantum transition.

To elicit most clearly the principles that operate, it is expedient to apply a twolevel approximation to the chromophores - an assumption that is fully justified if the donor and acceptor excited states are the lowest electronically excited levels of each chromophore, and other energy levels are of significantly higher energy. The rate of energy transfer between the two chromophores in a static electric field is again determined from the Fermi Rule. Here we define $\hat{\mathcal{E}}$ as the electric displacement unit vector of the static field (whose magnitude is $\mathcal{E}$ ), and $d^{\xi}=\mu^{e e(\xi)}-\mu^{00(\xi)}$ as the absolute difference between the magnitudes of the excited state $(e)$ and ground state static dipole-moments of chromophore $\xi$. For simplicity it is to be assumed that all static and transition dipole moments associated with a particular chromophore are parallel, although those associated with $D$ can have arbitrary orientation with respect to those associated with $A$. Introducing the shorthand $\boldsymbol{R}$ for $\boldsymbol{R}_{D A}$, the result for the rate is then cast as follows [16]:

$$
\begin{aligned}
W_{D A}= & \frac{9}{8 \pi c^{2} \tau_{D}} \times\left[S_{2}(\omega, \boldsymbol{R})\right. \\
& +\frac{\mathscr{\mathcal { E }}}{\hbar}\left\{\left|d^{D}\right|\left(\hat{\mathcal{E}} \cdot \hat{\boldsymbol{\mu}}^{D}\right)+\left|d^{A}\right|\left(\hat{\mathcal{E}} \cdot \hat{\boldsymbol{\mu}}^{A}\right)\right\} S_{1}(\omega, \boldsymbol{R}) \\
& +\frac{\mathcal{E}^{2}}{\hbar^{2}}\left\{\left[\left|d^{D}\right|\left(\hat{\mathcal{E}} \cdot \hat{\boldsymbol{\mu}}^{D}\right)+\left|d^{A}\right|\left(\hat{\mathcal{E}} \cdot \hat{\boldsymbol{\mu}}^{A}\right)\right]^{2}+2\left|d^{D}\right|\left|d^{A}\right|\left(\hat{\mathcal{E}} \cdot \hat{\boldsymbol{\mu}}^{D}\right)\left(\hat{\mathcal{E}} \cdot \hat{\boldsymbol{\mu}}^{A}\right)\right\} S_{0}(\omega, \boldsymbol{R}) \\
& -\frac{\mathscr{\mathcal { E }}^{3}}{\hbar^{3}}\left\{\left|d^{D}\right|\left|d^{A}\right|\left(\hat{\mathcal{E}} \cdot \hat{\boldsymbol{\mu}}^{D}\right)\left(\hat{\mathcal{E}} \cdot \hat{\boldsymbol{\mu}}^{A}\right)\left[\left|d^{D}\right|\left(\hat{\mathcal{E}} \cdot \hat{\boldsymbol{\mu}}^{D}\right)+\left|d^{A}\right|\left(\hat{\mathcal{E}} \cdot \hat{\boldsymbol{\mu}}^{A}\right)\right]\right\} S_{-1}(\omega, \boldsymbol{R}) \\
& \left.+\frac{\mathcal{E}^{4}}{\hbar^{4}}\left|d^{D}\right|^{2}\left|d^{A}\right|^{2}\left(\hat{\mathcal{E}} \cdot \hat{\boldsymbol{\mu}}^{D}\right)^{2}\left(\hat{\mathcal{E}} \cdot \hat{\boldsymbol{\mu}}^{A}\right)^{2} S_{-2}(\omega, \boldsymbol{R})\right] .
\end{aligned}
$$

Here the set of functions $S_{n}(\omega, \boldsymbol{R})$, defined by: 


$$
S_{n}(\omega, \boldsymbol{R})=\int F_{D}(\omega) \sigma_{A}(\omega) \omega^{n} \eta^{D A}(\omega, \boldsymbol{R}) \mathrm{d} \omega
$$

denote $\boldsymbol{R}$-dependent forms of frequency-weighted overlap integral. It is important to note that, in successive terms of Eq. (6), the powers of $\omega$ in the spectral overlap integral decrease. This indicates an increasing weighting towards the longwavelength end of the overlap between the donor emission and acceptor absorption curves, correlating with the order of nonlinearity in the dependence on $\mathcal{E}$. Figure 2 exhibits graphs derived from calculations of the transfer rate, using Eq. (6), based on typical values for the static field strength and transition dipole moments.

\section{Fig. 2}

Equation (6) can be understood as a Taylor series expansion in powers of $\mathscr{E}$, with the first term representing the normal rate of energy transfer (i.e. excluding static-field coupling), and with following terms signifying corrections of successively diminishing importance. The term describing energy transfer with one static interaction is the third term, and the last term describes energy transfer involving a coupling of both chromophores with the static field. The other terms represent quantum interference between the various pathways, of which the second term in Eq. (6) is linear in $\mathscr{E}$, and generally the most significant correction. However, if either the donor or acceptor transition is electric dipole-forbidden, the first and second terms of (6) vanish and the third term provides the leading rate contribution. If both the donor and acceptor transitions are electric dipole-forbidden, only the final term can provide any meaningful contribution to the rate. The physical significance is that energy may not transfer to or from such species without the presence of a static field (except, conceivably, through the involvement of a much weaker, higher order multipole moment). This emphasizes the potential significance of the static fieldinduced mechanism; within a suitably designed system it allows a switchable electric field to control the delivery of energy to the acceptor. 


\subsection{Influence of Throughput Radiation}

Pairwise energy transfer is influenced not only by the presence of static fields; it can also be modified by intense throughput laser radiation [17]. In this mechanism the radiation undergoes cooperative forward Rayleigh scattering by the donor-acceptor pair - effectively leaving the radiation unchanged, but either enhancing or diminishing the efficiency of energy transfer between the two chromophores. In the former, more interesting case the process is known as laser-assisted resonance energy transfer (LARET).

For simplicity, we restrict the following consideration to a system of non-polar (or only weakly polar) chromophores, again applying a two-level approximation. The laser-modified mechanism for energy transfer bears a degree of similarity to the static field-induced case described in sub-section 2.2, except that here a dynamic optical field is applied. Surprisingly, static dipoles of the donor and acceptor can still play a role, despite the oscillatory character of the field - but those which enter the rate expression now relate to electronically excited states. In other words, this mechanism again operates through shifts in the electron distributions, here associated with the donor decay and the acceptor excitation transitions. The total rate of energy transfer, in the presence of off-resonant laser light with a frequency $\omega^{\prime}$, is expressible as [17]; 


$$
\begin{aligned}
& W_{D A}= \frac{9}{8 \pi c^{2} \tau_{D}} \int F_{D}(\omega) \sigma_{A}(\omega) \omega^{2} \eta^{D A}(\omega, \boldsymbol{R}) \mathrm{d} \omega+\frac{9\left|\mu^{e e(D)} \| \mu^{e e(A)}\right| I\left(\omega^{\prime}\right)}{32 \pi \hbar^{2} c^{3} \varepsilon_{0} \omega^{\prime 2} \tau_{D}} \\
&+\left\{\frac { | \mu ^ { e e ( D ) } | | \mu ^ { e e ( A ) } | I ( \omega ^ { \prime } ) } { \hbar ^ { 2 } c \omega ^ { \prime 2 } \varepsilon _ { 0 } } \left[\left(\boldsymbol{e} . \hat{\boldsymbol{\mu}}^{D}\right)^{2}\left(\overline{\boldsymbol{e}} \hat{\boldsymbol{\mu}}^{A}\right)^{2} \int F_{D}(\omega) \sigma_{A}(\omega) \eta^{D A}\left(\omega_{+}, \boldsymbol{R}\right) \omega_{+}^{2} \mathrm{~d} \omega\right.\right. \\
&+\left(\overline{\boldsymbol{e}} \cdot \hat{\boldsymbol{\mu}}^{D}\right)^{2}\left(\boldsymbol{e} \cdot \hat{\boldsymbol{\mu}}^{A}\right)^{2} \int F_{D}(\omega) \sigma_{A}(\omega) \eta^{D A}\left(\omega_{-}, \boldsymbol{R}\right) \omega_{-}^{2} \mathrm{~d} \omega \\
&\left.+2\left[e^{\frac{2 i \omega^{\prime} R}{c}}+e^{-\frac{2 i \omega^{\prime} R}{c}}\right]\left(\boldsymbol{e} \cdot \hat{\boldsymbol{\mu}}^{D}\right)^{2}\left(\overline{\boldsymbol{e}}_{\cdot} \hat{\boldsymbol{\mu}}^{A}\right)^{2} \int F_{D}(\omega) \sigma_{A}(\omega) \eta^{D A}\left(\omega_{+}, \omega_{-}, \boldsymbol{R}\right)\left(\omega^{2}-\omega^{\prime 2}\right) \mathrm{d} \omega\right] \\
&-4\left[\left(\boldsymbol{e} . \hat{\boldsymbol{\mu}}^{D}\right)\left(\overline{\boldsymbol{e}} \cdot \hat{\boldsymbol{\mu}}^{A}\right)+\left(\overline{\boldsymbol{e}}_{.} \hat{\boldsymbol{\mu}}^{D}\right)\left(\boldsymbol{e} . \hat{\boldsymbol{\mu}}^{A}\right)\right]\left[e^{\frac{-i \omega^{\prime} R}{c}} \int F_{D}(\omega) \sigma_{A}(\omega) \eta^{D A}\left(\omega, \omega_{+}, \boldsymbol{R}\right) \omega \omega_{+} \mathrm{d} \omega\right. \\
&\left.\left.+e^{\frac{i \omega^{\prime} R}{c}} \int F_{D}(\omega) \sigma_{A}(\omega) \eta^{D A}\left(\omega, \omega_{-}, \boldsymbol{R}\right) \omega \omega_{-} \mathrm{d} \omega\right]\right\}
\end{aligned}
$$

In the above, $\omega_{ \pm}$represents $\omega \pm \omega^{\prime} ; I\left(\omega^{\prime}\right)$ is the irradiance of the laser radiation and $\boldsymbol{e}$ is the corresponding polarization vector ( $\overline{\boldsymbol{e}}$ being its complex conjugate, the distinction allowing for circular or elliptical polarizations). The first three $\eta$ factors are as described previously, with $\omega \pm \omega^{\prime}$ substituted for $\omega$ where appropriate. The other $\eta$ factors arise from interference terms and have a slightly more complicated form:

$$
\eta^{D A}\left(\omega_{1}, \omega_{2}, \boldsymbol{R}\right)=\frac{c^{6}}{\omega_{1}^{3} \omega_{2}^{3} R^{6}} \kappa_{3}^{2}+\frac{c^{4}}{\omega_{1}^{2} \omega_{2}^{2} R^{4}} \kappa_{3}^{2}-\left[\frac{c^{4}}{\omega_{1}^{3} \omega_{2} R^{4}}+\frac{c^{4}}{\omega_{1} \omega_{2}^{3} R^{4}}\right] \kappa_{3} \kappa_{1}+\frac{c^{2}}{\omega_{1} \omega_{2} R^{2}} \kappa_{1}^{2}
$$

where $\omega_{1}$ and $\omega_{2}$ stand for $\omega, \omega_{+}$or $\omega_{-}$as required by Eq. (8).

The conventional (field-free) mechanism for RET is dominant at incident intensities below about $10^{13} \mathrm{~W} \mathrm{~m}^{-2}$. At intensities of around $10^{16} \mathrm{~W} \mathrm{~m}^{-2}$ - relatively high, but easily produced using pulsed lasers - a rate enhancement of $10 \%$ or more can be introduced by the LARET mechanism, even for non-polar systems, and using 
significantly off-resonant frequencies. However for chromophores which are both polar and chiral, additional terms arise [17], and these can lead to rate enhancements as high as $30 \%$ or more. Moreover, by judiciously tuning the laser in regions close to optical absorption bands, the detailed frequency dependence of the spectral overlap integrals in Eq. (8), and the distance-dependent factors of (9), can be exploited to effect even greater levels of enhancement. Finally, due to the linear and quadratic dependencies on irradiance for the LARET rate contributions, any further increase in the intensity of the throughput laser light can also be employed to generate a favorably disproportionate increase in the energy transfer rate. We believe that the use of off-resonant laser light to augment energy transfer should find many applications in artificial systems, since it provides an easily controlled, switchable process.

\section{Energy Transfer in Multi-Chromophore Environments}

The previous section has described energy transfer at the fundamental level in terms of a coupling between two electronically isolated chromophores. In reality, however, most systems comprise numerous chromophores, and the vast majority of these systems are specifically non-homogenous - or else comprise chemically similar chromophores distributed in non-homogeneous electronic environments. In the overall migration of energy, from the site of its initial deposition to the site of its chemical action, the spectroscopic gradient is one of the key directional principles obviating random diffusion. Despite each RET step occurring without energy loss, a small amount of energy is lost after each transfer step, in the form of vibrational relaxation (ultimately manifest as heat). Consequently, each species when acting as acceptor receives energy associated with a longer optical wavelength than its donor

initially acquired. This causes back-transfer to be very inefficient, due to the poorer overlap of the acceptor emission and donor absorption spectra, enhancing a flow of energy towards acceptors with increasingly longer wavelength absorption profiles. The operation of the spectroscopic gradient on RET is shown in figure 3. 


\section{Fig. 3}

Since each transfer event may take place in an electronic environment modified by one or more other chromophores, in proximity to the interaction pair, it is necessary to entertain the possible role of a 'third body' $M$ in effecting other changes to the character of pairwise energy transfer. For example, in a dendrimeric system the third body might be another chromophore of the same type as either donor or acceptor, in the same general vicinity. In a photosynthetic complex the third body could be an ancillary pigment, or even one residue of a support protein unit. The involvement of such species as an influence on the rate of donor-acceptor transfer has received surprisingly little attention, yet it transpires that surrounding chromophores, especially any that are strongly polar, can substantially affect the rate of energy transfer without themselves changing state. This mechanism can be represented in general terms as follows;

$$
D^{*}+A+M \longrightarrow D+A^{*}+M
$$

\section{Fig. 4}

Associated with this mechanism, depicted in Figure 4 (and in addition to the quantum amplitude for direct transfer not involving $M$, as described in the initial section) three distinct contributions to the amplitude can be identified: a static dipole of $M$ interacts with either $D$ or $A$, or a dynamic dipole of $M$ acts as an intermediary for the energy transfer. In a sense the static interaction is comparable to that described in the last sub-section, except that here the field is produced in situ rather than being externally delivered. The total rate of energy transfer between two chromophores in the presence of a third body is [18]; 


$$
\begin{aligned}
& W_{D A}=\frac{9}{8 \pi c^{2} \tau_{D}} \int F_{D}(\omega) \sigma_{A}(\omega) \omega^{2} \eta^{D A}\left(\omega, \boldsymbol{R}_{D A}\right) \mathrm{d} \omega+\frac{9}{128 \pi^{3} \hbar^{2} c^{2} \varepsilon_{0}^{2} \tau_{D}} \\
& \times\left\{\left[\frac{\left|d^{D}\right|^{2}\left(\kappa_{3}^{D M}\right)^{2}}{R_{D M}^{6}}+\frac{\left|d^{A}\right|^{2}\left(\kappa_{3}^{A M}\right)^{2}}{R_{A M}^{6}}+\frac{\left|d^{D}\right|\left|d^{A}\right| \kappa_{3}^{D M} \kappa_{3}^{A M}}{R_{D M}^{3} R_{A M}^{3}}\right]\left|\mu^{M}\right|^{2} \int F_{D}(\omega) \sigma_{A}(\omega) \eta^{D A}\left(\omega, \boldsymbol{R}_{D A}\right) \mathrm{d} \omega\right. \\
& +\hbar^{2} \int F_{D}(\omega) \sigma_{A}(\omega)\left|\alpha^{M}(\omega)\right|^{2} \omega^{2} \eta^{D M}\left(\omega, \boldsymbol{R}_{D M}\right) \eta^{A M}\left(\omega, \boldsymbol{R}_{A M}\right) \mathrm{d} \omega \\
& +\left[\frac{\left|d^{D}\right| \kappa_{3}^{D M}}{R_{D M}^{3}}+\frac{\left|d^{A}\right| \kappa_{3}^{A M}}{R_{A M}^{3}}\right] 4\left|\mu^{M}\right| \hbar \int F_{D}(\omega) \sigma_{A}(\omega) \omega \eta^{D A}\left(\omega, \boldsymbol{R}_{D A}\right) \mathrm{d} \omega \\
& +\left[\frac{\left|d^{D}\right| \kappa_{3}^{D M}}{R_{D M}^{3}}+\frac{\left|d^{A}\right| \kappa_{3}^{A M}}{R_{A M}^{3}}\right] \frac{\left|\mu^{M}\right| \hbar c^{6}}{R_{D A}^{3} R_{D M}^{3} R_{A M}^{3}} \int F_{D}(\omega) \sigma_{A}(\omega)\left|\alpha^{M}(\omega)\right| \omega^{-5} \chi\left(\omega, \boldsymbol{R}_{D A}, \boldsymbol{R}_{D M}, \boldsymbol{R}_{A M}\right) \mathrm{d} \omega \\
& \left.+\frac{4 \pi \varepsilon_{0} \hbar^{2} c^{6}}{R_{D A}^{3} R_{D M}^{3} R_{A M}^{3}} \int F_{D}(\omega) \sigma_{A}(\omega)\left|\alpha^{M}(\omega)\right| \omega^{-4} \chi\left(\omega, \boldsymbol{R}_{D A}, \boldsymbol{R}_{D M}, \boldsymbol{R}_{A M}\right) \mathrm{d} \omega\right\}
\end{aligned}
$$

where $\mu^{M}$ and $\alpha^{M}$ are, respectively, the static dipole moment and the dynamic polarizability (at frequency $\omega$ ) of $M$ and, as defined previously, $d^{\xi}$ is the difference between the static excited state and ground state dipole-moments of chromophore $\xi$. Additionally, $\chi\left(\omega, \boldsymbol{R}_{D A}, \boldsymbol{R}_{D M}, \boldsymbol{R}_{A M}\right)$ is given by;

$$
\left.\mathfrak{R} e\left\{\left(1-\frac{i \omega R_{D A}}{c}\right) \kappa_{3}^{D A}-\frac{\omega^{2} R_{D A}^{2}}{c^{2}} \kappa_{1}^{D A}\right]\left[\left(1+\frac{i \omega R_{D M}}{c}\right) \kappa_{3}^{D M}-\frac{\omega^{2} R_{D M}^{2}}{c^{2}} \kappa_{1}^{D M}\right]\left[\left(1+\frac{i \omega R_{A M}}{c}\right) \kappa_{3}^{A M}-\frac{\omega^{2} R_{A M}^{2}}{c^{2}} \kappa_{1}^{A M}\right]\right\}
$$

Note again the variable frequency-weighting of the various spectral overlap terms. Unless at least one of $D, A$ and $M$ is polar, the only viable three-body process is where $M$ plays the role of an electronic bridge - in this case most of the terms in Eq. (11) vanish, with only the first, third and final terms providing any contribution to the rate of energy transfer. The largest influence of $M$ occurs when it is polar, consistent with the interpretation that it thereby effects a distortion in the donor and/or acceptor electron distributions. Equally, (as opposed to the case of LARET), even if the donor and acceptor are non-polar, they may acquire induced moments through the influence of $M$. 
Previous research into the influence on energy transfer [18] has shown that the third body chromophore has a significant effect on the rate of energy transfer only when that chromophore is very close to either one of the donor-acceptor pair, typically at distances less then $1.5 \mathrm{~nm}$. In this region, the third-body influence typically ranges from a $50 \%$ enhancement of the rate to a $40 \%$ reduction, depending on the arrangement of the chromophores involved. The exact rate distribution with respect to position depends on the relative dipole moment orientation of the three chromophores, but in general the influence of $M$ is greatest when the dipole is positioned just outside wavefunction overlap of $D$ or $A$. An example of this is given in Figure 5. Considering the potential magnitude of the rate modification cited above, it is clearly imperative to take this mechanism into account when analyzing systems comprising a large number of closely spaced chromophores, such as most lightharvesting materials.

\section{Fig. 5}

\section{Directed Energy Transfer Systems}

\subsection{Light-Harvesting Complexes}

In the realm of plant science, directional RET is well known to be an extremely significant process in the operation of photosynthetic units. In order to most effectively utilize the sunlight that falls on them, all photosynthetic organisms have a system of antenna complexes surrounding the reaction centers where photosynthesis takes place. The photosystems of purple bacteria, such as Rhodopseudomonas acidophila and Rhodobacter sphaeroides [19], have been most extensively studied [20-22] by means of ultrashort pulsed laser spectroscopy [23], X-ray protein crystallography [24], as well as hole-burning and absorption studies [25]. In such systems bacteriochlorophyll is the key light-harvesting pigment. Two complexes, LH1 and LH2, form coplanar ring structures, in which the bacteriochlorophyll 
pigments are present as dimers, their local protein environment determining their precise wavelength of maximum absorption. The complex LH1, which surrounds the reaction center $(\mathrm{RC})$, is composed of $\mathrm{B} 880$, a bacteriochlorophyll dimer with an absorption maximum at $880 \mathrm{~nm}$. These structures ostensibly exhibit formation as a ring when studied independently, though some studies have suggested that the ring is not closed when the RC is present $[21,26]$. The LH1 complex is in turn surrounded by a number of $\mathrm{LH} 2$ complexes, comprising both $\mathrm{B} 850$ rings above and $\mathrm{B} 800$ rings below.

The antenna complexes absorb sunlight and the acquired energy migrates towards the reaction centre by a series of short-range, radiationless energy transfer steps. Although the energy initially absorbed by most chlorophylls is associated with shorter wavelength photons than is eventually received by the reaction centre, some 'red chlorophylls' absorb at longer wavelength than the RC, broadening the absorption profile of the complex [27]. Energy absorbed by outlying complexes is quickly and efficiently directed towards the reaction center - a process often referred to as channeling or funneling - due to the operation of a spectroscopic gradient between the antenna complexes and the reaction center. Not only does this allow an organism to harvest light incident on a large surface area but, by pooling energy from a large number of antenna chromophores, energy of a higher equivalent frequency can be produced. This is essential, since the majority of the incident light from the sun has too low a frequency for its individual photons to effect chemical photosynthesis.

It is not only spectroscopic properties of the chromophores that determine the direction of energy flow; the chromophore positioning and orientation are also important. Two-dimensional optical spectroscopy can unveil the intricate interplay between spectral and spatial overlap features in light-harvesting complexes, as has been beautifully exhibited in recent studies on the Fenna-Olsen-Matthews bacteriochlorophyll $a$ protein of green sulfur bacteria [28]. Interrogating the system with a sequence of ultrashort laser pulses, the optical response of the sample can be interpreted to reveal linear absorption processes as well as couplings between chromophores, and dynamical aspects of the energy transfer. The results show that excitation relocation does not simply proceed by stepwise transfer from one energy state to another of nearest energy - it depends on strong coupling between 
chromophores, determined by the extent of their spatial overlap. Thus, excitation relocation may involve fewer intermediary chromophores than might otherwise be expected.

\subsection{Dendrimers}

The efficiency of photosynthetic units - especially the photobacterial systems optimized for operation at very low levels of intensity - has encouraged the design of a variety of synthetic light-harvesting systems that mimic their efficient energy funneling properties. The materials which have received most attention are dendrimers - macromolecules consisting of molecular units repeatedly branching out from a central core designed to act as an excitation trap [29, 30]. The branching functionalization of terminal groups leads to successive generations of structures, each with an increased number of peripheral antenna chromophores. In ideal cases the requisite spectroscopic gradient is established through chemically similar chromophores situated in generationally different locations, and having different electronic properties due to subtly differing chemical environments. This arrangement expedites an emulation of the energy funneling observed in natural lightharvesting systems. In both cases peripheral chromophores absorb incident photons and the excitation energy relocates across the structure [31-33], ultimately being funneled into the core [34]. Striking examples of this principle can be seen in classic work on perylene-functionalised phenylacetylene dendrimers $[35,36]$. An example of a organosilicon cationic dendrimer is given in figure 6.

\section{Fig. 6}

More recent work on dendrimers has seen a number of variations on the basic structural theme. As an example, dendrimers with dye molecules embedded in their internal cavities have proven to have very high energy harvesting efficiencies (approximately $80 \%$ in one case, where eosin is embedded in a dendrimer with sixtyfive chromophore groups of four different types [38]). Other dendrimeric structures include: a molecular square, twenty-chromophore unit, which exhibits biomimetic energy transfer from outer to inner (pyrene to perylene) chromophores [39]; and 
multi-porphyrin systems [40] - in which energy transfer from a zinc porphyrin to its free base counterpart is enhanced by the presence of suitable bridging chromophores [41]. Other work has focused on the synthesis and characterization of multiporphyrin arrays based on a phenylethynyl proto-dendrimeric framework [42], and dendritic chains $[43,44]$ expediting directional energy transfer along their length (a concept holding promise for the design of nanoelectronic devices).

A particularly striking success is an artificial photosynthetic antenna-reaction center complex comprising four zinc tetraarylporphyrins covalently linked, through phenylethynyl dendrites, to a free base porphyrin-fullerene acting as a 'reaction center'. Following photoexcitation of the peripheral zinc porphyrin (antenna), energy migrates to the central zinc porphyrin (donor) from which it transfers to free base porphyrin (acceptor), initiating electron transfer to the fullerene (reaction centre). In this system, the charge-separated excited state is generated with an impressively high quantum yield of 0.90 , based on the light absorbed by the zinc porphyrin antenna [45, 46]. Energy harvesting dendrimers are increasingly being developed for use as organic light-emitting diode materials [47-50]. Furthermore, energy pooling porphyrin dendrimers have begun to find an application in photodynamic therapy (PDT) as photosensitisers [51-53] - energy harvesting here leading to the targeted photochemical destruction of cancer cells via the generation of singlet oxygen.

\subsection{Energy Transfer between Dipole Arrays}

One of the operational principles under consideration for new optical switching devices is to configure a resonant coupling of throughput optical radiation to matter with a suitable absorption profile. Here the key requirement is to enable optically generated excitation to propagate between particles with a suitably matching frequency response. Many of the proposed systems involve internal transfers of electronic excitation - for example, electron transfer activated by an applied electric field [54-56] or laser-induced RET [57-61]. Such devices hold significant promise for the furtherance of ultrafast communication and signal processing systems. Within this context, and as one example of directed energy transfer, we now explore a nanoscale optical switching concept based on LARET. 
We consider a scheme for the use of pulsed laser light to selectively channel energy between corresponding pixels in two parallel planar arrays. The concept is based on a configuration in which the transfer of energy from any excited donor to a counterpart acceptor in the opposing plane would be forbidden by conventional RET, but for which a quantum channel is opened by throughput laser radiation. For its physical implementation a pair of two-dimensional arrays can be envisaged, each consisting of equally spaced, identical chromophores arranged on a square lattice. The dipole transition moment of any given chromophore is parallel to all other transition moments within the same plane; each donor in one layer has a counterpart acceptor, with an orthogonal transition moment, in the other layer. For any single excited donor pixel, resonance energy transfer to its counterpart pixel is forbidden since the $\kappa$ factor for that transfer is equal to zero. However, the application to the system of laser radiation, with an appropriate intensity, frequency and polarization, allows energy to transfer between the two pixels, via a LARET pathway as described previously. A diagram of this system is given in figure 7. By engineering the separation of the layers, it can be ensured that energy transfer is always directed from any specific donor to its corresponding acceptor. In passing we note that, although electrical field influences are also capable of activating forbidden energy transfer, affording another possible basis for such an application, LARET proves to be the most amenable to controlled nanoscale implementation.

\section{Fig. 7}

To produce a meaningful system, energy transfer from a given donor to its counterpart acceptor must be greatly favorable compared to any involving another acceptor or even another donor - in other words, cross-talk has to be minimized. A detailed analysis of the geometric and orientational features of the system and associated constraints has been given elsewhere [62]. The analysis reveals that the probability of transfer to the intended acceptor strongly depends on the aspect ratio the relative magnitude of the lattice spacing and the spatial separation of the arrays as well as the intensity of the applied laser radiation. Figure 8 illustrates this with a particularly suitable relative positioning - where the spatial separation is one-tenth that of the lattice spacing. Energy can be directed to the intended acceptor on 
application of laser light with an irradiance in the region of $10^{16} \mathrm{~W} \mathrm{~m}^{-2}$, for example. The favorability of this configuration is illustrated by the fact that a larger aspect ratio of 1:2 would result in a vast increase in the energy transfer probability to unintended acceptors, compromising the fidelity of the system. The appraisal of other methods for directing energy transfer in this and related systems, and potential device applications, are the subjects of ongoing research in the quantum electrodynamics group at UEA.

\section{Fig. 8}

\section{Future Applications}

In this chapter a variety of means for effecting directed energy transfer have been discussed and key results from the quantum theory have been presented. It has also been illustrated, by reference to several very different systems, how the principles can be implemented. Each mechanism offers distinct opportunities for future applications, which are being brought closer to fruition as new photonic technology is developed. The principle of directed electronic energy transfer is especially amenable to nanophotonic device implementation, since it enables the propagation of electronic energy to be specifically directed over sub-wavelength dimensions.

The best known mechanism for introducing a vector character to the flow of energy in a complex medium is a spectroscopic gradient. As has been shown, this requires a serendipitous correlation between the progression of quantum energy levels and the geometric layout of successive chromophore types in a multi-chromophore system. The exploitation of this principle, which operates extremely effectively in natural light-harvesting complexes, requires extraordinary skills in the design and synthesis of man-made biomimetic analogues, the best current examples of which are found in dendrimeric polymers. It now appears that the application of an electric field offers opportunities to effect further control over the flow of energy in such structures.

In device applications, the static field mechanism appears to require levels of field that would be most easily sustained in microscopic or sub-microscopic domains 
(obviating the extremely high voltages that would otherwise be required), and these may perhaps be best achieved through surface field effects. Rapidly switching a directing field offers further intriguing possibilities. To achieve such control at the molecular level, utilizing local fields generated by neighboring dipoles, allows the necessary fields to be produced more readily - but such a system is less amenable to real-time experimental control. Here one can envisage composite materials with a molecular architecture designed to deliver optically acquired energy from antenna chromophores to suitable traps, expedited by the incorporation of strongly polar groups.

It appears to be the case of optically induced RET switching that the most promising opportunities for device implementation may arise. It is highly significant that the laser systems capable of delivering the necessary levels of irradiance are precisely those that also offer directly controllable, ultrafast speeds of switching. The parallel processing possibilities which arise with array implementation suggest a variety of optical interconnect applications - including flat panel displays and optical communications routing technology. The realization of such applications should be viewed in the context of ongoing advances in nanolithographic fabrication, which are driving optical technology to ever greater levels of speed and miniaturization. The mechanisms for controlling energy flow are only just beginning to receive attention, and it will be fascinating to observe how materials science and nanotechnology will rise to the challenge of exploiting the new opportunities which they open up.

\section{Acknowledgements}

Research in the quantum electrodynamics group at UEA is funded by the UK Engineering and Physical Sciences Research Council (EPSRC). We gladly acknowledge the award of an EPSRC studentship to RGC. 


\section{References}

1. Förster T (1948) Annalen Der Physik 2: 55

2. Craig DP, Thirunamachandran T (1986) Accounts of Chemical Research 19: 10

3. Andrews DL, Sherborne BS (1987) Journal of Chemical Physics 86: 4011

4. Juzeliunas G, Andrews DL (2000) Advances in Chemical Physics 112: 357

5. Daniels GJ, Jenkins RD, Bradshaw DS, Andrews DL (2003) Journal of Chemical Physics 119: 2264

6. Salam A (2005) Journal of Chemical Physics 122: 044112

7. Salam A (2005) Journal of Chemical Physics 122: 044113

8. Dexter DL (1953) Journal of Chemical Physics 21: 836

9. Scholes GD, Ghiggino KP, Oliver AM, Paddon-Row MN (1993) Journal of the American Chemical Society 115: 4345

10. Yeow EKL, Haines DJ, Ghiggino KP, Paddon-Row MN (1999) Journal of Physical Chemistry A 103: 6517

11. Yeow EKL, Ghiggino KP (2000) Journal of Physical Chemistry A 104: 5825

12. Smith TA, Lokan N, Cabral N, Davies SR, Paddon-Row MN, Ghiggino KP (2002) Journal of Photochemistry and Photobiology a-Chemistry 149: 55

13. Forde TS, Hanley QS (2005) Photochemical \& Photobiological Sciences 4: 609

14. Juzeliunas G, Andrews DL (1999) In: Andrews DL, Demidov AA (eds) Resonance Energy Transfer. Wiley, Chichester p 65

15. Wieb van der Meer B (1999) In: Andrews DL, Demidov AA (eds) Resonance Energy Transfer. Wiley, Chichester p 151

16. Andrews DL, Bittner AM (1993) Journal of Luminescence 55: 231

17. Allcock P, Jenkins RD, Andrews DL (2000) Physical Review A 6102: 023812

18. Daniels GJ, Andrews DL (2002) Journal of Chemical Physics 117: 6882(E)

19. Hu X, Schulten K (1998) Biophysical Journal 75: 683

20. Sundström V, Pullerits T, van Grondelle R (1999) Journal of Physical Chemistry B 103: 2327

21. Roszak AW, Howard TD, Southall J, Gardiner AT, Law CJ, Isaacs NW, Cogdell RJ (2003) Science 302: 1969

22. Krueger BP, Scholes GD, Gould IR, Fleming GR (1999) PhysChemComm: 8

23. Katiliene Z, Katilius E, Uyeda GH, Williams JC, Woodbury NW (2004) Journal of Physical Chemistry B 108: 3863

24. Hu X, Damjanovik A, Ritz T, Schulten K (1998) Proceedings of the National Academy of Science USA 95: 5935

25. Wu H-M, Rätsep M, Jankowiak R, Cogdell RJ, Small GJ (1998) Journal of Physical Chemistry B 102: 4023

26. Jungas C, Ranck JL, Rigaud JL, Joliot P, Vermeglio A (1999) Embo Journal 18: 534 
27. Sener MK, Schulten K (2005) In: Andrews DL (ed) Energy Harvesting Materials. World Scientific, Singapore p 1

28. Brixner T, Stenger J, Vaswani HM, Cho M, Blankenship RE, Fleming GR (2005) Nature 434: 625

29. Bar-Haim A, Klafter J (1998) Journal of Luminescence 76-7: 197

30. Bar-Haim A, Klafter J (1998) Journal of Physical Chemistry B 102: 1662

31. Tretiak S, Chernyak V, Mukamel S (1998) Journal of Physical Chemistry B 102: 3310

32. Swallen SF, Shi ZY, Tan WH, Xu ZF, Moore JS, Kopelman R (1998) Journal of Luminescence 76-7: 193

33. Yeow EKL, Ghiggino KP, Reek JNH, Crossley MJ, Bosman AW, Schenning A, Meijer EW (2000) Journal of Physical Chemistry B 104: 2596

34. Adronov A, Frechet JMJ (2000) Chemical Communications: 1701

35. Devadoss C, Bharathi P, Moore JS (1996) Journal of the American Chemical Society 118: 9635

36. Shortreed MR, Swallen SF, Shi ZY, Tan WH, Xu ZF, Devadoss C, Moore JS, Kopelman R (1997) Journal of Physical Chemistry B 101: 6318

37. Aulenta F, Hayes W, Rannard S (2003) European Polymer Journal 39: 1741

38. Hahn U, Gorka M, Vögtle F, Vicinelli V, Ceroni P, Maestri M, Balzani V (2002) Angewandte Chemie-International Edition 41: 3595

39. Würthner F, Sautter A (2003) Organic \& Biomolecular Chemistry 1: 240

40. Van Patten PG, Shreve AP, Lindsey JS, Donohoe RJ (1998) Journal of Physical Chemistry B 102: 4209

41. Kilså K, Kajanus J, Mårtensson J, Albinsson B (1999) Journal of Physical Chemistry B 103: 7329

42. Brodard P, Matzinger S, Vauthey E, Mongin O, Papamicaël C, Gossauer A (1999) Journal of Physical Chemistry A 103: 5858

43. Cho HS, Song NW, Kim YH, Jeoung SC, Hahn S, Kim D, Kim SK, Yoshida N, Osuka A (2000) Journal of Physical Chemistry A 104: 3287

44. Tamura M, Gao D, Ueno A (2001) Chemistry-a European Journal 7: 1390

45. Kuciauskas D, Liddell PA, Lin S, Johnson TE, Weghorn SJ, Lindsey JS, Moore AL, Moore TA, Gust D (1999) Journal of the American Chemical Society 121: 8604

46. Kodis G, Liddell PA, de la Garza L, Clausen PC, Lindsey JS, Moore AL, Moore TA, Gust D (2002) Journal of Physical Chemistry A 106: 2036

47. Halim M, Samuel IDW, Pillow JNG, Burn PL (1999) Synthetic Metals 102: 1113

48. Freeman AW, Koene SC, Malenfant PRL, Thompson ME, Frechet JMJ (2000) Journal of the American Chemical Society 122: 12385

49. Anthopoulos TD, Markham JPJ, Namdas EB, Lawrence JR, Samuel IDW, Lo SC, Burn PL (2003) Organic Electronics 4: 71

50. Furuta P, Brooks J, Thompson ME, Frechet JMJ (2003) Journal of the American Chemical Society 125: 13165 
51. Stapert HR, Nishiyama N, Jiang DL, Aida T, Kataoka K (2000) Langmuir 16: 8182

52. Nishiyama N, Stapert HR, Zhang GD, Takasu D, Jiang DL, Nagano T, Aida T, Kataoka K (2003) Bioconjugate Chemistry 14: 58

53. Zhang GD, Harada A, Nishiyama N, Jiang DL, Koyama H, Aida T, Kataoka K (2003) Journal of Controlled Release 93: 141

54. Aviram A (1988) Journal of the American Chemical Society 110: 5687

55. Chen J, Reed MA, Rawlett AM, Tour JM (1999) Science 286: 1550

56. Yang ZQ, Lang ND, Di Ventra M (2003) Applied Physics Letters 82: 1938

57. Alvaro M, Chretien MN, Ferrer B, Fornes V, Garcia H, Scaiano JC (2001) Chemical Communications: 2106

58. Just EM, Wasielewski MR (2000) Superlattices and Microstructures 28: 317

59. Sangu S, Kobayashi K, Shojiguchi A, Kawazoe T, Ohtsu M (2003) Journal of Applied Physics 93: 2937

60. Wada O (2004) New Journal of Physics 6: 183

61. Ham BS (2001) Etri Journal 23: 106

62. Andrews DL, Crisp RG (in press) Journal of Fluorescence

63. Andrews DL, Crisp RG (in press) Journal of Optics A-Pure and Applied Optics 


\section{Figure Legends}

Figure 1: Depictions of: $(a)$ resonance energy transfer; $(b)$ electric field induced energy transfer; $(c)$ laser-assisted resonance energy transfer. In each case, energy transfers from the donor on the left to the acceptor on the right; in the latter two cases an auxiliary influence affects the rate of transfer.

Figure 2: $\quad$ Influence of a static field on energy transfer as the polarization vector of the static field is rotated relative to the transfer pair. (a) The donor and acceptor transition moments are parallel, and the static field strength is $10^{10} \mathrm{~V} \mathrm{~m}^{-1}$. (b) The donor and acceptor transition moments are antiparallel to each other and the static field strength is $10^{11} \mathrm{~V} \mathrm{~m}^{-1}$.

Figure 3: $\quad$ Energy level depiction illustrating the directing influence of the spectroscopic gradient on resonance energy transfer. Boxes indicate ground and excited electronic manifolds. Following excitation of donor $D$, energy transfers to the acceptor $A$. The dotted lines represent a range of vibrational energy levels within each electronic manifold, encompassed in the emission and absorption profiles of the chromophores. The strong overlap of the donor emission and acceptor absorption spectra, as denoted by the shaded area in the upper graph (wavelength increasing to the right), indicates that energy transfer is favorable in this instance. After the transfer, intramolecular vibrational relaxation (IVR) occurs in the acceptor, shifting its emission spectrum to a slightly longer wavelength than its absorption counterpart. Since the donor absorption profile is associated with a slightly higher frequency than its emission spectrum (again due to IVR), back-transfer from $A$ to $D$ is prohibited by the much weaker overlap of their respective emission and absorption profiles, as shown in the lower graph against wavelength. Both graphs are drawn on the same scale.

Figure 4: Depiction of third-body assisted energy transfer. Energy transfers from the donor on the left to the acceptor on the right, with the supporting chromophore exerting either a static or dynamic influence on the transfer pair.

Figure 5: Influence of a neighboring dipole $M$ on the rate of energy transfer between two chromophores, here located at coordinates $(2.5,2.0)$ and $(5.5,2.0)$ (positions measured in nm). Both chromophore transition moments are aligned parallel to the $y$-axis, the ancillary dipole is oriented at an angle of $\pi / 2$ to the $y$-axis. The influence of $M$ increases as the color darkens from orange to red, with the darkest shade of red indicating a $200 \%$ increase of the rate compared to the lightest areas. White 
denotes positions of significant wavefunction overlap, where a different (exchange) mechanism would come into play.

Figure 6: Example of a polycationic dendrimer. The core is surrounded by a repeatedly branching structure terminating in peripheral antenna groups. Reprinted with permission from reference [37].

Figure 7: $\quad$ Arrays of donors (upper array) and acceptors (lower array) aligned with orthogonal dipole transition moments. Throughput laser radiation enables energy transfer from the excited donor (shown in white in the diagram) to its counterpart acceptor. The separation between the arrays is exaggerated for clarity. Reprinted with permission from reference [62].

Figure 8: $\quad$ Energy transfer from a single excited donor to an array of acceptors in the absence (left) and presence (right) of throughput laser radiation. The separation between the donor and acceptor arrays is one-tenth that of the lattice spacing of each array, and an irradiance of $10^{16} \mathrm{~W} \mathrm{~m}^{-2}$ is sufficient to direct energy transfer. The vertical scale represents the efficiency of energy transfer to the chromophore at that position in the array, black dots representing the position of each acceptor. 